\title{
Minuscule Heaps Over Dynkin diagrams of type $\tilde{A}$
}

\author{
Manabu HAGIWARA * \\ Institute of Industrial Science, University of Tokyo \\ e-mail: manau@imailab.iis.u-tokyo.ac.jp
}

Submitted: Oct 18, 2002; Accepted: Dec 12, 2003; Published: Jan 2, 2004

\begin{abstract}
A minuscule heap is a partially ordered set, together with a labeling of its elements by the nodes of a Dynkin diagram, satisfying certain conditions derived by J. Stembridge. This paper classifies the minuscule heaps over the Dynkin diagram of type $\tilde{A}$.
\end{abstract}

\section{Introduction}

The aim of this paper is to classify the minuscule heaps over a Dynkin diagram of type $\tilde{A}$.

Let $A$ be a symmetrizable generalized Cartan matrix, and let $\mathfrak{g}$ be a corresponding Kac-Moody Lie algebra. Let $\Gamma$ be a Dynkin diagram which is an encoding of $A$. Minuscule heaps arose in connection with $\lambda$-minuscule elements of the Weyl group $W$ of $\mathfrak{g}$. According to Proctor [9] and Stembridge [12] the notion of $\lambda$-minuscule elements of $W$ was defined by Peterson in his unpublished work in the 1980's. For an integral weight $\lambda$, an element $w$ of $W$ is said to be a $\lambda$-minuscule element if it has a reduced decomposition $s_{i_{1}} s_{i_{2}} \ldots s_{i_{r}}$ such that

$$
s_{i_{j}} s_{i_{j+1}} \ldots s_{i_{r}} \lambda=s_{i_{j+1}} \ldots s_{i_{r}} \lambda-\alpha_{i_{j}}(1 \leq \forall j \leq r),
$$

and it is called minuscule if $w$ is $\lambda$-minuscule for some integral weight $\lambda$. Here $\alpha_{i}$ is the simple root corresponding to $s_{i}$. It is known that a minuscule element is fully commutative, namely any reduced decomposition can be converted into any other by exchanging adjacent commuting generators several times (see $[9, \S 15],[10$, Theorem A] and [11, Theorem 2.2], or [12, Proposition 2.1]). To a fully commutative element $w$, one can associate a $\Gamma$-labeled poset called its heap.

A $\Gamma$-labeled poset is a triple $(P, \leq, \phi)$ in which $(P, \leq)$ is a poset and $\phi: P \rightarrow N(\Gamma)$ is any map (called the labeling map). A linear extension of a $\Gamma$-labeled poset naturally

\footnotetext{
*The author's name is "HAGIWARA Manabu" by Japanese ordering.
} 
determines a word in the generators of $W$. The heap of the fully commutative element $w$ is a $\Gamma$-labeled poset whose linear extensions determine all reduced decompositions of $w$. A minuscule heap is the heap of a minuscule element of $W$.

Stembridge obtained certain structural conditions for a finite $\Gamma$-labeled poset to be a minuscule heap ([11, Proposition 3.1]). In this paper a minuscule heap is defined by a finite $\Gamma$-labeled poset which satisfies the conditions (H1-1), (H1-2) and (H2a) (see §2). In the following, we state a relation between minuscule elements and minuscule heaps.

Let $(P, \leq, \phi)$ be a minuscule heap. Put $r:=\# P$. Let $\mu: P \rightarrow[1, r]$ be a linear extension of $P$, namely $\mu$ is a bijection and if $p \leq q$ then $\mu(p) \leq \mu(q)$. For $\mu$, we associate a minuscule heap $(P, \leq, \phi)$ to $w \in W$ by the following expression

$$
w:=s_{\phi \circ \mu^{-1}(1)} s_{\phi \circ \mu^{-1}(2)} \ldots s_{\phi \circ \mu^{-1}(r)} .
$$

We note that an element obtained from a minuscule heap, by the relation above, is a minuscule element. Conversely for any minuscule element $w$ there exists a unique minuscule heap which determines $w$.

In [11], there is an important condition which states that "the labels that occur in $P$ index an acyclic subset of the Dynkin diagram". A nice consequence of this condition is that if it holds then $P$ is a ranked poset. However Dynkin diagrams $\Gamma$ of type $\tilde{A}$ are cyclic, and most of minuscule heaps over $\Gamma$ are not ranked. In this paper, we introduce an analogy of slant lattices [6] (here called $\left.L_{k}\right)(\S 8)$ and use it to prove that a subset $P$ of an extended slant lattice $L_{k}$ is a convex subset if and only if $P$ is a minuscule heap over $\Gamma$ up to isomorphic. Slant lattices $L$ were also used to classify the minuscule heaps over simply-laced, star-shaped Dynkin diagrams in [6].

It is known that the affine permutation group $\tilde{S}_{n+1}$ is isomorphic to an affine Weyl group $W\left(\tilde{A}_{n}\right)$. In [5], Green showed that the 321-avoiding permutations of affine permutations coincide with the fully commutative elements of $W\left(\tilde{A}_{n}\right)$. He showed also that the fully commutative elements of $W\left(\tilde{A}_{n}\right)$ form a union of Kazhdan-Lusztig cells. Here we show that the fully commutative elements of $W\left(\tilde{A}_{n}\right)$ coincide with its minuscule elements [Theorem 5.1].

The paper is organized as follows. In $\S 2$ we recall and provide some basic terminology. In $\S \S 3,4$ we collect some general facts on poset and on $\Gamma$-labeled poset with a general Dynkin diagram. In $\S 5$, we show that the fully commutative elements of $W(\tilde{A})$ coincide with the minuscule elements of $W(\tilde{A})$. From $\S 4$, we characterize the minuscule heaps over a Dynkin diagram of type $\tilde{A}$. In $\S 6$, we characterize the totally ordered minuscule heaps over a Dynkin diagram of type $\tilde{A}$. In $\S 7$, we determine the structure of a subposet which we call $v$-interval. In $\S 8$, we characterize the minuscule heaps over a Dynkin diagram of type $\tilde{A}$ up to isomorphism and introduce the notion of extended slant lattices $L_{k}$. In $\S 9$, we show that any minuscule heap over a Dynkin diagram of type $\tilde{A}$ is isomorphic to a convex subset of an extended slant lattice $L_{k}$. 


\section{Definitions}

We start with the definition of general terms associated to a partially ordered set. We denote the number of elements of a set $P$ by $\# P$.

Let $(P, \leq)$ be a poset (partially ordered set). For $p, q \in P$, we say that $q$ covers $p$ (or $p$ is covered by $q$ ) if $p<q$ and $(p, q)=\emptyset$, and denote by $p \rightarrow q$. We say that $p, q$ are a covering pair if $p \rightarrow q$ or $q \rightarrow p$. In this paper, we assume that $P$ is completely determined by the covering relations, namely

(*) If $p, q \in P$ and $p \leq q$ then there exists a finite sequence of elements of $P$, say $p_{0}, p_{1}, \ldots, p_{r}$ such that $p_{0}=p, p_{r}=q$ and $p_{i}$ covers $p_{i-1}$ for $1 \leq i \leq r$.

We call such a sequence $p_{0}, p_{1}, \ldots, p_{r}$ a saturated chain from $p$ to $q$.

We denote ordering relations on posets as follows. Let $P$ be a poset and let $Q$ be its subset. For $x, y \in Q$, we write $[x, y]_{P}=\left\{z \in P \mid x \leq_{P} z \leq_{P} y\right\}$ and write $[x, y]_{Q}=\{z \in$ $\left.Q \mid x \leq_{Q} z \leq_{Q} y\right\}$. In general, a maximal connected subposet of $P$ is called a connected component of $P$. A subset $Q$ of $P$ is said to be convex in $P$ if whenever $p, q \in Q$ and $p \leq q$ we have $[p, q]_{P} \subset Q$.

Let $\Gamma$ be a Dynkin diagram and let $N(\Gamma)$ be the node set of $\Gamma$. By an abuse of language, we sometimes identify $N(\Gamma)$ with $\Gamma$. We say that a triple $(P, \leq, \phi)$ (or simply $P)$ is a $\Gamma$-labeled poset if $(P, \leq)$ is a partially ordered set, and $\phi$ is any map from $P$ to $N(\Gamma)$. We call $\phi$ the labeling map and call $\phi(p)$ the label of $p$. We denote $\operatorname{Im} \phi$ by supp $P$, and call it the support of $P$. For each $v \in N(\Gamma)$, we put

$$
P_{v}:=\{p \in P \mid \phi(p)=v\}
$$

For $v \in N(\Gamma)$ and $p, q \in P$ satisfying $p<q$, we say that $[p, q]$ is a $v$-interval if $p, q \in P_{v}$ and $(p, q) \cap P_{v}=\emptyset$.

Let $(P, \leq, \phi),(Q, \preceq, \psi)$ be $\Gamma$-labeled posets. We say that $P$ and $Q$ are isomorphic as $\Gamma$-labeled poset if there exists a poset isomorphism $\Phi: P \rightarrow Q$ such that $\phi(p)=$ $\psi(\Phi(p))(\forall p \in P)$.

Let $\Gamma$ and $\Gamma^{\prime}$ be Dynkin diagrams, and let $\left(a_{i, j}\right)_{i, j \in I},\left(a_{i, j}^{\prime}\right)_{i, j \in I^{\prime}}$ be the corresponding generalized Cartan matrices. Let $P$ be a $\Gamma$-labeled poset and let $Q$ be a $\Gamma^{\prime}$-labeled poset. We say that $P$ and $Q$ are abstractly isomorphic (or isomorphic if no confusion arises) if there is a poset isomorphism $\alpha: P \rightarrow Q$ and an isomorphism of subdiagrams $\beta$ : supp $P \rightarrow \operatorname{supp} Q$ (namely a bijection $\operatorname{supp} P \rightarrow \operatorname{supp} Q$ such that $a_{i, j}=a_{\beta(i), \beta(j)}^{\prime}$ for all $i, j \in \operatorname{supp} P)$ such that $\beta$ maps the label of $p$ to the label of $\alpha(p)$ for every $p \in P$.

Let $D(3):=\{w, x, y, z\}$ be a set and let $\rightarrow$ be a binary relation on $D(3)$ with $w \rightarrow$ $x, x \rightarrow z, w \rightarrow y, y \rightarrow z$. Let $\leq$ be an ordering on $D(3)$ which is the reflective, transitive closure of $\rightarrow$. Let $\Gamma$ be the Dynkin diagram of type $A_{3}$ and let $N(\Gamma)=\{1,2,3\}$ be the node set of $\Gamma$. (Put 1,2,3 on $N(\Gamma)$ from an edge node to another one.) Define a map $\phi: D(3) \rightarrow N(\Gamma)$ by putting $\phi(w)=\phi(z):=2, \phi(x):=1$ and $\phi(y):=3$. We regard $D(3)$ as a $\Gamma$-labeled poset with $\phi$. Let $\Gamma^{\prime}$ be a Dynkin diagram. We say that $\Gamma^{\prime}$-labeled poset $Q$ is a diamond if $Q$ and $(D(3), \leq, \phi)$ are abstractly isomorphic. 
Let $A=\left(a_{i, j}\right)_{i, j \in N(\Gamma)}$ be a Cartan matrix corresponding to $\Gamma$. We say that a $\Gamma$-labeled poset $(P, \leq, \phi)$ is a minuscule heap if $P$ is finite and satisfies the conditions (H1-1),(H1-2) and $(\mathrm{H} 2 \mathrm{a})$.

(H1-1) For $p, q \in P$, if $p \rightarrow q$, then $\phi(p)$ and $\phi(q)$ are either equal or adjacent in $\Gamma$.

(H1-2) For $p, q \in P$, if $\phi(p)$ and $\phi(q)$ are either equal or adjacent in $\Gamma$ then $p$ and $q$ are comparable.

(H2a) For $p, q \in P$, if $\phi(p)=\phi(q)$ and $p \leq q$ then $\sum_{x \in[p, q]} a_{\phi(x), \phi(p)}=2$.

In particular we regard an empty set as a minuscule heap.

Remark 2.1. In [11], Stembridge obtained two structural conditions, which he called (H1) and (H2), for a finite $\Gamma$-labeled poset to be a minuscule heap. In this paper we separate (H1) to two conditions (H1-1),(H1-2). And we use the condition (H2a) instead of (H2) which are equivalent. (Proposition 4.4.)

\section{Basic Properties on Poset}

In this section we provide some general facts on posets. We omit the proofs below since they are straightforward.

Proposition 3.1. Let $S$ be a set and let $\leadsto$ be a binary relation on $S$. Let $\preceq$ be the reflexive, transitive closure of $\leadsto$.

Then $(S, \preceq)$ is a poset if and only if $s=s_{0} \leadsto s_{1} \leadsto \cdots \leadsto s_{r}=s$ implies $s_{0}=s_{1}=$ $\cdots=s_{r}=s$ for some $r \geq 0$, where $s, s_{0}, s_{1}, \ldots, s_{r} \in S$.

Proposition 3.2. Let $S, \leadsto, \preceq$ be the same as Proposition 3.1. Assume that $(S, \preceq)$ be a poset.

Then $\leadsto$ is the covering relation on $(S, \preceq)$ if and only if $p \leadsto q$ and $p=p_{0} \leadsto p_{1} \leadsto$ $\cdots \leadsto p_{r}=q$ implies $r=1$.

Proposition 3.3. Let $(P, \leq)$ be a poset and let $G$ be a group which acts on $P$ as a poset automorphism, namely $p \leq q$ if and only if $g(p) \leq g(q)$ for $g \in G$ and $p, q \in P$.

Assume that $G$ satisfies the following condition,

- for $p \in P$ and $g \in G$, if $p$ and $g(p)$ are comparable then $p=g(p)$.

Put $P / G:=\{\bar{p} \mid p \in P\}$, where $\bar{p}=\{g(p) \mid g \in G\}$, and put a relation $\preceq$ on $P / G$ as following,

$$
\bar{p} \preceq \bar{q} \text { if and only if } p \leq g(q) \text { for some } g \in G(\bar{p}, \bar{q} \in G / P) \text {. }
$$

Then it follows,

- $\preceq$ is well-defined,

- $(P / G, \preceq)$ is a poset. 
Proposition 3.4. Let $P$ be a poset and let $p, q \in P$ satisfying $p \leq q$. Then $[p, q]$ is a convex subset of $P$.

Proposition 3.5. Let $P$ be a poset and $Q$ be a convex subset. If $z$ is a minimal or maximal element of $Q$ then $Q \backslash\{z\}$ is a convex subset of $P$.

\section{Basic Properties on $\Gamma$-labeled Posets}

In this section we provide some general facts on $\Gamma$-labeled posets over a general Dynkin diagram. (See $[7]$ or $[8]$ for the definition of Dynkin diagrams.)

Proposition 4.1. Let $(P, \leq, \phi)$ be a $\Gamma$-labeled poset. If $(P, \leq, \phi)$ satisfies (H1-2) then $P_{v}$ is a totally ordered set for each $v \in N(\Gamma)$.

Proof. By (H1-2), $p$ and $q$ are comparable, where $p, q \in P_{v}$.

Proposition 4.2. Let $(P, \leq, \phi)$ be a $\Gamma$-labeled poset which satisfies (H1-1) and (H1-2). Then $P$ is connected if and only if supp $P$ is connected.

Proof. Assume that $P$ is connected. For $u, v \in \operatorname{supp} P$, there exists $p \in P_{v}$ and $q \in P_{u}$. Now we can take a sequence $p=p_{0}, p_{1}, \ldots, p_{r}=q$ such that $p_{i-1}, p_{i}$ are a covering pair. Then $\phi\left(p_{0}\right), \phi\left(p_{1}\right), \ldots, \phi\left(p_{r}\right)$ consists of a connected subdiagram of $\Gamma$ by (H1-1). Hence supp $P$ is connected.

Conversely assume that $\operatorname{supp} P$ is connected. Let $p, q \in P$ and put $v:=\phi(p), u:=$ $\phi(q)$. Since $\Gamma$ is connected, we can take a sequence $v=v_{0}, v_{1}, \ldots, v_{r}=u \in \operatorname{supp} P$ such that $v_{i-1}$ and $v_{i}$ are adjacent in $\Gamma$. Take some $p_{i} \in P_{v_{i}}(1 \leq i \leq r-1)$ and put $p_{0}=p, p_{r}=q$. Then $p_{i-1}, p_{i}$ are comparable by (H1-2). So $P$ is connected.

Proposition 4.3. Let $(P, \leq, \phi)$ be a $\Gamma$-labeled poset satisfying (H1-1) and (H1-2). Let $P_{1}, P_{2}, \ldots, P_{r}$ be the connected components of $P$. Then supp $P=\sqcup_{i=1}^{r} \operatorname{supp} P_{i}$. In particular $v$ and $u$ are distinct and non-adjacent, where $v \in \operatorname{supp} P_{i}, u \in \operatorname{supp} P_{j}$ and $i \neq j$.

Proof. If there exists $v \in \operatorname{supp} P_{i} \cap \operatorname{supp} P_{j}$ then we can take $p \in P_{i} \cap P_{v}$ and $q \in$ supp $P_{j} \cap P_{v}$. By (H1-2), $p$ and $q$ are comparable. This implies $i=j$ because $P_{i}, P_{j}$ are connected components.

If there exists $v \in \operatorname{supp} P_{i}$ and $u \in \operatorname{supp} P_{j}$ such that $v$ and $u$ are adjacent in $\Gamma$ then we obtain a contradiction by a similar argument.

Proposition 4.4. Let $(P, \leq, \phi)$ be a finite $\Gamma$-labeled poset. If $(P, \leq, \phi)$ satisfies (H1-1) and (H1-2) then each of the following each conditions are equivalent to (H2a).

(H2) For any v-interval $[p, q]$, we have $\sum_{x \in[p, q]} a_{\phi(x), \phi(p)}=2$.

(H2b) For any $v$-interval $[p, q]$, we have $\sum_{x \in(p, q)} a_{\phi(x), \phi(p)}=-2$. 
Proof. By $a_{v, v}=2$, it is obvious that (H2) and (H2b) are equivalent for any $v \in N(\Gamma)$. It is also obvious that (H2a) implies (H2).

We assume that (H2) holds. By (H1-2), $P_{v}$ is a totally ordered set. Let $p, q$ be elements of $P_{v}$ which satisfy $p \leq q$. If we have $p=q$ then (H2a) trivially holds, since $a_{v, v}=2$ for $v \in N(\Gamma)$. So we assume $p<q$. Let $p=p_{0}, p_{1}, \ldots, p_{r}=q$ be the elements of $P_{v} \cap[p, q]$ by an increasing ordering. For $x \in[p, q] \backslash\left(\cup_{1 \leq i \leq r}\left[p_{i-1}, p_{i}\right]\right), x$ and $p_{j}$ are incomparable for some $0 \leq j \leq r$. By $(\mathrm{H} 1-2), \phi(x)$ is different from $v$ and not adjacent to $v$. Thus we have $a_{\phi(x), v}=0$. This implies

$$
\sum_{x \in[p, q]} a_{\phi(x), v}=\sum_{1 \leq i \leq r} \sum_{x \in\left[p_{i-1}, p_{i}\right)} a_{\phi(x), v}+a_{\phi\left(p_{r}\right), v}=0+2=2 .
$$

Remark 4.5. Let $\Gamma$ be a simply-laced Dynkin diagram and let $v \in N(\Gamma)$. Let $[p, q]$ be a $v$-interval. (H2b) requires that there exists just two elements of $(p, q)$ whose labels are adjacent to $v$ in $\Gamma$. This fact is very important since the Dynkin diagram of type $\tilde{A}_{n}(n \geq 2)$ is simply-laced. However we investigate a minuscule heap over the Dynkin diagram of type $\tilde{A}_{1}$. In this case, (H2b) requires that there exists only one element of $(p, q)$ whose labels are adjacent to $v$ in $\Gamma$. These facts are used to prove Propositions 4.6, 6.1 and 6.2 .

Proposition 4.6. Let $\Gamma$ be a simply-laced Dynkin diagram and let $(P, \leq, \phi)$ be a minuscule heap over $\Gamma$. Let $p, q \in P$ such that $\phi(p)=\phi(q)$. If there exists an element $x \in P$ such that $p \rightarrow x \rightarrow q$ then $[p, q]$ is a $\phi(p)$-interval. In particular $[p, q]$ is a diamond.

Proof. We note that $\phi(x)$ and $\phi(p)$ are adjacent in $\Gamma$. If there exists $y \in(p, q)$ such that $\phi(y)=\phi(p)$ then $x$ and $y$ are comparable by (H1-2). This implies that we have either $p<x<y$ or $y<x<q$. It contradicts $p \rightarrow x \rightarrow q$. So we have $(p, q) \cap P_{\phi(p)}=\emptyset$. However there exists $y \in(p, q)$ such that $\phi(y)$ is adjacent to $\phi(p)$ by Remark 4.5. Let $p=p_{0}, p_{1}, \ldots, p_{r}=q$ be a saturated chain from $p$ to $q$ which contains $y$. We note that this saturated chain does not contain $x$. In fact a sequence $p, x, q$ is a unique saturated chain which contains $x$.

For $p=p_{0}, p_{1}, \ldots, p_{r}=q, y$ is the only element which can cover $p$, and $y$ is the only element which can be covered by $q$. Thus this saturated chain consists of $p, y, q$. Hence we have $[p, q]=\{p, x, y, q\}$. By (H1-1) and (H2), $[p, q]$ is a diamond.

Proposition 4.7. Let $\Gamma$ be a Dynkin diagram and let $\Phi$ be a graph automorphism on $\Gamma$. If a $\Gamma$-labeled poset $(P, \leq, \phi)$ is a minuscule heap then $(P, \leq, \Phi \circ \phi)$ is a minuscule heap. Furthermore these minuscule heaps are abstractly isomorphic.

Proof. Since $\Phi$ is a graph automorphism, it is obvious that (H1-1) and (H1-2) hold. As $a_{v, u}=a_{\Phi(v), \Phi(u)}$, (H2a) holds. 


\section{Relation Between Fully Commutative Elements and Minuscule Elements}

First we show that the fully commutative elements of $W(\tilde{A})$ coincide with its minuscule elements.

Theorem 5.1. Let $\Gamma$ be a simply-laced Dynkin diagram with a finite node set. The fully commutative elements of $W(\Gamma)$ coincide with its minuscule elements if and only if $\Gamma$ is of type $A$ or $\tilde{A}$.

Proof. It is well known that a minuscule element is fully commutative.

Assume $\Gamma$ branches off. Then there exists a node $v \in N(\Gamma)$ such that the number of the adjacent nodes to $v$ is larger than two. Let $x, y, z$ be adjacent nodes to $v$. We can verify that $s_{v} s_{x} s_{y} s_{z} s_{v}$ is fully commutative, where $s_{u}$ is a generator associated to $u \in N(\Gamma)$. But it violates $(\mathrm{H} 2)$ and so it would not be minuscule. Hence $\Gamma$ cannot have a junction. Thus $\Gamma$ must be of type $A$ or $\tilde{A}$.

If $\Gamma$ is of type $A$, then it is well-known that a fully commutative element is minuscule. The remaining case is when $\Gamma$ is of type $\tilde{A}$. Let $w$ be a fully commutative element of $W(\tilde{A})$ and let $s_{1} s_{2} \ldots s_{r}$ be a reduced expression of $w$. By the commutativity of $w$, if $s_{i}, s_{j}$ are consecutive occurrences of the generator $s$ (meaning that $s_{i}=s_{j}=s$ for some generator $s(i<\mathrm{j})$ and $s_{i} \neq s$ for $\left.i<h<j\right)$, then there are at least two generators $s_{h_{1}}, s_{h_{2}}$ such that $s_{i}$ and $s_{h_{1}}$ (or $s_{h_{2}}$ ) are non-commutative $i<h_{1}, h_{2}<j$. If there is no consecutive occurrences of any generators, then $w$ is minuscule.

Remember that any heap of a fully commutative element satisfies (H1). For proving that $w$ is minuscule, it is sufficient that there exists just two non-commutative generators $s_{h_{1}}, s_{h_{2}}$. If not, we can take three non-commutative generators from $s_{i+1}, \ldots, s_{j-1}$. Now, as $\Gamma$ is of type $\tilde{A}$, each node has only two adjacent nodes. Thus we can take consecutive occurrences $s_{i^{\prime}}, s_{j^{\prime}}$ of $s^{\prime}$. By the commutativity of $w$, we can take two generators $s_{h_{1}^{\prime}}, s_{h_{2}^{\prime}}$ from $s_{i^{\prime}+1}, \ldots, s_{j^{\prime}-1}$ which are non-commutative to $s^{\prime}$. The nodes associated to $s_{i^{\prime}+1}, s_{j^{\prime}+1}$ are adjacent to the node associated to $s^{\prime}$ and they are different from $s_{i}$ because $s_{i} \neq s_{h}$ for $i<h<j$. This implies $s_{i^{\prime}+1}=s_{j^{\prime}+1}$, in other words they are consecutive occurrences. By using a similar argument, the length of $w$ must be infinite. It cannot happen.

\section{Totally Ordered Minuscule Heaps over Dynkin Di- agrams of Type $\tilde{A}$}

In this section we determine the structure of totally ordered minuscule heaps over Dynkin diagrams of type $\tilde{A}$. From this section on we assume that $\Gamma$ is a Dynkin diagram of type $\tilde{A}_{n}$ with the node set $N(\Gamma):=\{0,1, \ldots, n\}$. (see Figure 1 for the definition of Dynkin diagram of type $\tilde{A}_{n}$.) We associate $i \in \mathbb{Z}$ to $j \in N(\Gamma)=\{0,1, \ldots, n+1\}$ by the following rule $j=i \bmod (n+1)$. We note that the Dynkin diagram of type $\tilde{A}_{1}$ and its Cartan matrix $A:=A(\Gamma)$ are different from others of type $\tilde{A}_{n}(n \geq 2)$. First we classify the minuscule heaps over $\tilde{A}_{1}$. In fact a minuscule heap over $\Gamma\left(\tilde{A}_{1}\right)$ is a totally ordered set. 


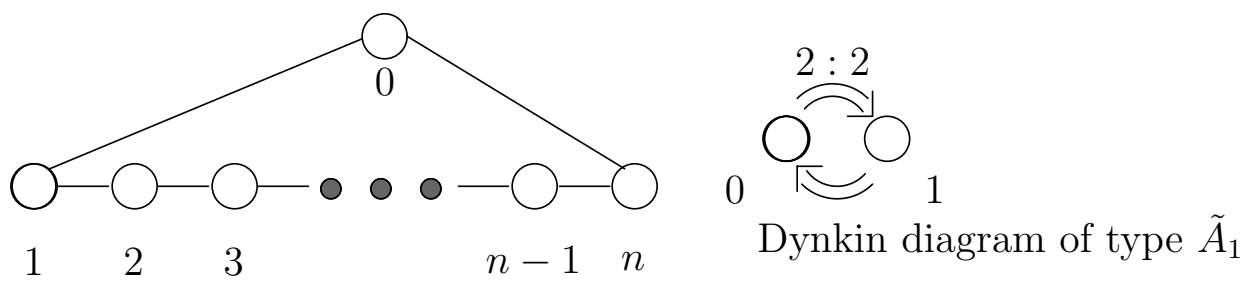

Dynkin diagram of type $\tilde{A}_{n}(n \geq 2)$

Figure 1: The Dynkin diagram of type $\tilde{A}_{n}$

The Cartan matrix $A=A\left(\Gamma\left(\tilde{A}_{1}\right)\right)=\left(a_{i, j}\right)$ is

$$
A:=\left(\begin{array}{cc}
a_{0,0} & a_{0,1} \\
a_{1,0} & a_{1,1}
\end{array}\right)=\left(\begin{array}{cc}
2 & -2 \\
-2 & 2
\end{array}\right) .
$$

Proposition 6.1. Let $\Gamma$ be the Dynkin diagram of type $\tilde{A}_{1}$. A minuscule heap over $\Gamma$ is a totally ordered set and is characterized by $r:=\# P$ and the label $v$ of its smallest element if $r \neq 0$. Namely, define a $\Gamma$-labeled poset $(P, \leq, \phi)$ by putting

- $P=\left\{p_{1}, p_{2}, \ldots, p_{r}\right\}$,

- $p_{i-1} \rightarrow p_{i}(1<i \leq r)$,

- $\leq$ is the transitive and reflective closure of $\rightarrow$,

- $\phi\left(p_{i}\right) \equiv v+i-1(\bmod 2)$.

Then $(P, \leq, \phi)$ is a minuscule heap over $\Gamma$. Conversely a minuscule heap over $\Gamma$ is abstractly isomorphic to a minuscule heap as defined above.

Proof. Define $P$ as above. Then it is obvious that (H1-1) and (H1-2) hold on $P$. By the definition of $P$, a $v$-interval has the form $\left[p_{i}, p_{i+2}\right]$ for $v \in N(\Gamma)$. So we have

$$
\sum_{x \in\left(p_{i}, p_{i+2}\right)} a_{\phi(x), v}=\sum_{x \in\left\{p_{i+1}\right\}} a_{\phi(x), v}=-2 .
$$

This implies that $P$ is a minuscule heap.

Conversely assume that $(P, \leq, \phi)$ is a minuscule heap. Then it is obvious that $P$ is a totally ordered set by the shape of $\Gamma$ and (H1-2). Hence we can write $P=\left\{p_{1}, p_{2}, \ldots, p_{r}\right\}$ with $p_{i-1} \rightarrow p_{i}(1<i \leq r)$. By (H2b), we have $\phi\left(p_{i-1}\right) \neq \phi\left(p_{i}\right)$. Since the labels of $p_{i}$ are binary values $(\{0,1\})$, two nodes always alternatively appear. Thus we have $\phi\left(p_{i}\right) \equiv v+i-1(\bmod 2)$.

By a similar argument above, we can determine the structure of a totally ordered minuscule heap over $\Gamma\left(\tilde{A}_{n}\right)(n \geq 2)$. 
Proposition 6.2. Let $(P, \leq, \phi)$ be a totally ordered minuscule heap over $\Gamma$. Put $P=$ $\left\{p_{0}, p_{1}, \ldots, p_{r}\right\}$ with $p_{i-1} \rightarrow p_{i}(1 \leq i \leq r)$. Then the labels of the elements of $P$ are either of type (T1) or (T2),

(T1) $\phi\left(p_{i}\right) \equiv \phi\left(p_{0}\right)+i(\bmod n+1)$

(T2) $\phi\left(p_{i}\right) \equiv \phi\left(p_{0}\right)-i(\bmod n+1)$

Proof. By (H2b), we have $\phi\left(p_{0}\right) \neq \phi\left(p_{1}\right)$. By (H1-1), we have either $\phi\left(p_{1}\right)=\phi\left(p_{0}\right)+1$ or $\phi\left(p_{1}\right)=\phi\left(p_{0}\right)-1$.

Assume $\phi\left(p_{1}\right)=\phi\left(p_{0}\right)+1$. Then we have either $\phi\left(p_{2}\right)=\phi\left(p_{1}\right)+1$ or $\phi\left(p_{2}\right)=\phi\left(p_{1}\right)-1$. If we have $\phi\left(p_{2}\right)=\phi\left(p_{1}\right)-1$ then $\phi\left(p_{2}\right)=\phi\left(p_{0}\right)$. By Proposition 4.6, $\left[p_{0}, p_{2}\right]$ is a $\phi\left(p_{0}\right)$ interval. However we have

$$
\sum_{x \in\left(p_{0}, p_{2}\right)} a_{\phi(x), \phi\left(p_{0}\right)}=\sum_{x \in\left\{p_{1}\right\}} a_{\phi(x), \phi\left(p_{0}\right)}=-1 \neq-2 .
$$

It contradicts $(\mathrm{H} 2 \mathrm{~b})$. Thus we have $\phi\left(p_{2}\right)=\phi\left(p_{1}\right)+1$. By repeating an argument above, the labels of the elements are of type (T1).

By a similar argument, we obtain the case (T2) from the assumption $\phi\left(p_{1}\right)=\phi\left(p_{0}\right)-$ 1.

We say that $P$ is of type (T1) (resp. (T2)) if the labels of $P$ are of type (T1) (resp. (T2)).

\section{$7 \quad$ The Structure of $v$-intervals}

In this section we investigate the structure of $v$-intervals for a minuscule heap over $\Gamma\left(\tilde{A}_{n}\right)$ with $n \geq 2$. To determine $v$-intervals for $v \in N(\Gamma)$ is useful to determine the structure of a minuscule heap.

By the symmetry of the shape of the Dynkin diagram of type $\tilde{A}$, to determine the structure of all of 1-intervals is equivalent to determine that of all of $v$-intervals for any $v \in N(\Gamma)$. Hence we investigate the structure of the 1-intervals.

Lemma 7.1. Let $P$ be a minuscule heap over $\Gamma\left(\tilde{A}_{n}\right)$ and let $\left[p_{1}, q_{1}\right] \subset P$ be a 1-interval. Let $p_{2}, q_{2}$ be elements of $\left(p_{1}, q_{1}\right)$ whose labels are adjacent to 1 in $\Gamma$. Then we have $\phi\left(p_{2}\right) \neq \phi\left(q_{2}\right)$.

Proof. We note that $\phi\left(p_{2}\right), \phi\left(q_{2}\right)$ must be 0 or 2 . Our claim is that $\phi\left(p_{2}\right)=\phi\left(q_{2}\right)$ is impossible.

If we have $\phi\left(p_{2}\right)=\phi\left(q_{2}\right)=2$ then $p_{2}$ and $q_{2}$ are comparable. Let us assume that $p_{2}<q_{2}$ then $\left[p_{2}, q_{2}\right]$ is a 2-interval by (H2b). Hence there exist $p_{3}, q_{3} \in\left(p_{2}, q_{2}\right)$ such that $p_{2} \rightarrow p_{3}, q_{3} \rightarrow q_{2}$ and $\phi\left(p_{3}\right), \phi\left(q_{3}\right)$ are adjacent to 2 . Now $\phi\left(p_{3}\right), \phi\left(q_{3}\right)$ can be only equal to 1 or 3 . However they cannot be equal to 1 since $\left[p_{1}, q_{1}\right]$ is a 1 -interval. So we have $\phi\left(p_{3}\right)=\phi\left(q_{3}\right)=3$. By repeating the arguments above, we can take a 0 -interval $\left[p_{n+1}, q_{n+1}\right]$ from $\left(p_{1}, q_{1}\right)$ and we know that $\left[p_{n+1}, q_{n+1}\right]$ must contain an element whose label is 1 . It contradicts that $\left[p_{1}, q_{1}\right]$ is a 1 -interval. 
For $i=1,2,3$, we say that a 1 -interval $[p, q]$ is of type ( $V i)$ if $[p, q]$ satisfies the following:

(V1) $[p, q]$ is a totally ordered set and consists of $n+2$ elements. The labels of the elements in the increasing order are $1,2,3, \ldots, n-1, n, 0,1$ respectively;

(V2) $[p, q]$ is a totally ordered set and consists of $n+2$ elements; The labels of the elements in the increasing order are $1,0, n, n-1, \ldots, 3,2,1$ respectively.

(V3) $[p, q]$ is a diamond.

Proposition 7.2. Any 1-interval $[p, q]$ is either of type (V1), (V2) or (V3).

Proof. By Lemma 7.1, $(p, q)$ contains a unique pair of elements $x, y$ whose labels are 0,2 respectively. By (H1-1), only $x$ or $y$ can cover $p$ and only $x$ or $y$ can be covered by $q$.

Assume that both $x$ and $y$ cover $p$ then we claim that $[p, q]$ is of type (V3). By the assumption that $x$ and $y$ are incomparable, there is a saturated chain $x=p_{1}, p_{2}, \ldots, p_{r}=q$ from $x$ to $q$ which does not contain $y$. We note that $\phi\left(p_{r-1}\right)$ is either 0 or 2 , namely $p_{r-1}$ is either $x$ or $y$. This implies $p_{r-1}=x$ and $r=2$. Thus we have $x \rightarrow q$. By a similar argument, we have $y \rightarrow q$. So $[p, q]=\{p, x, y, q\}$ is of type (V3).

Next assume that only $x$ covers $p$. We claim that $[p, q]$ is of type (V1). Let $p=p_{0}, x=$ $p_{1}, p_{2}, \ldots, p_{r}=q$ be a saturated chain from $p$ to $q$. We note that $\phi\left(p_{i-1}\right)-\phi\left(p_{i}\right)$ is either 1 or -1 because $\phi\left(p_{i-1}\right), \phi\left(p_{i}\right)$ are adjacent. If these labels are all different then they are of type (V1) or (V2). If there are repeated labels then we can take a pair $p_{i}, p_{i+2}$ such that $\phi\left(p_{i}\right)=\phi\left(p_{i+2}\right)$. Let us choose such a minimal $i$. By Prop. 4.6, $\left[p_{i}, p_{i+2}\right]$ is a diamond. Thus there exists $p_{i+1}^{\prime} \in\left[p_{i}, p_{i+2}\right]$ such that $\phi\left(p_{i+1}^{\prime}\right)$ is not equal to $\phi\left(p_{i}\right)$ and $\phi\left(p_{i+1}\right)$. If we change $p_{i+1}$ to $p_{i+1}^{\prime}$ then we take another saturated chain $p_{0}, p_{1}, \ldots, p_{i+1}^{\prime}, \ldots, p_{r}$ from $p$ to $q$ such that $\phi\left(p_{i-1}\right)=\phi\left(p_{i+1}^{\prime}\right)$. By using the same argument, there exists a saturated chain $p=p_{0}, x=p_{1}, p_{2}, \ldots, p_{r}=q$ with $\phi\left(p_{1}\right)=\phi\left(p_{3}\right)$. It contradicts that $[p, q]$ is a 1-interval. So each labels are different. There exists only one saturated chain from $p$ to $q$ is only $p_{0}, p_{1}, \ldots, p_{r}$. Assume that there exists another saturated chain $p=q_{0}, x=q_{1}, \ldots, q_{r}=q$. So, there exists $q_{i}$ such that $q_{i} \neq p_{i}$ and $q_{j}=p_{j}(0 \leq j<i)$. By the above argument, we have $\phi\left(p_{i}\right)=\phi\left(q_{i}\right)$. By (H1-2), $p_{i}$ and $q_{i}$ are comparable. If $p_{i}<q_{i}$ then we have $q_{i-1}<p_{i}<q_{i}$. If $q_{i}<p_{i}$ then we have $p_{i-1}<q_{i}<p_{i}$. These are contradictions. So $[p, q]$ is of type (V1).

By using a similar argument, if we assume that only $y$ covers $p$ then we obtain that $[p, q]$ is of type (V2).

For not only 1-intervals but also for a $v$-interval $[p, q]$, we say that $[p, q]$ is of type (Vi) if $[p, q]$ satisfies the following:

(V1) $[p, q]$ is a totally ordered set and consists of $n+2$ elements. The labels of the elements in the increasing order are $v, v+1, v+2, \ldots, v+n-1, v+n, v$ respectively;

(V2) $[p, q]$ is a totally ordered set and consists of $n+2$ elements. The labels of the elements in the increasing order are $v, v+n, v+n-1, \ldots, v+2, v+1, v$ respectively;

(V3) $[p, q]$ is a diamond. 
Define a graph automorphism $\Phi: \Gamma \rightarrow \Gamma$ by $\Phi(i):=i+1(\bmod n+1)$. If we apply $\Phi$ and Prop. 4.7 to Prop. 7.2 then we can characterize all the 2-intervals. Thus we characterize all the $v$-intervals for all $v \in N(\Gamma)$.

Proposition 7.3. Any v-interval $[p, q]$ is either of type (V1), (V2) or (V3).

Proposition 7.4. If $P$ contains a v-interval of type (V1) (resp. (V2)) for some $v \in N(\Gamma)$ then $P$ is a totally ordered set and is of type (V1) (resp (V2)).

Proof. Let $(P, \leq, \phi)$ be a minuscule heap over $\Gamma\left(\tilde{A}_{n}\right)$ which contains a $v$-interval $[p, q]$ of type (V1). It is sufficient to show that every element of $P$ covers and is covered by at most one of its element. If we assume that there exists $x \in P$ which covers or is covered by two (or more) elements, then we get a contradiction as follows. By supp $[p, q]=N(\Gamma)$, $x$ is comparable to an element $p \in[p, q]$. Thus we have $p<x$ or $x<q$. Now we assume $p<x$ holds. (If we can consider the dual poset $P^{*}$, we can verify the case $x<q$.)

Assume $x \in P$ is covered by two (or more) elements $x_{1}, x_{2}$ of $P$. We can take a saturated chain $p=p_{0} \rightarrow p_{1} \rightarrow \cdots \rightarrow p_{i}=x$. By the shape of $\Gamma, \phi\left(x_{1}\right)$ or $\phi\left(x_{2}\right)$ is equal to $\phi\left(p_{i-1}\right)$. Without loss of generality, we can assume that $\phi\left(x_{1}\right)=\phi\left(p_{i-1}\right)$. By Prop. 4.6, $\left[p_{i-1}, x_{1}\right]$ is a diamond. Thus $p_{i-1}$ must be covered by two (or more) elements. By repeating this argument, $p$ is covered by two (or more) elements. We denote these elements by $p_{1}, p_{1}^{\prime}$. Since $\phi\left(p_{1}^{\prime}\right)$ is next to $\phi(p)$, we have $\phi\left(p_{1}^{\prime}\right)=\phi(q)$. So $p_{1}^{\prime}, q$ are comparable. Because $p_{1}^{\prime}$ covers $p$, we have $p_{1}^{\prime}<q$. On the other hand, $p_{1}^{\prime}$ is not contained in $[p, q]$, a contradiction.

Assume $x \in P$ covers two (or more) elements $x_{1}, x_{2}$ of $P$. We can take two saturated chains $p=p_{0} \rightarrow \cdots \rightarrow p_{i-1}=x_{1} \rightarrow p_{i}=x$ and $p=q_{0} \rightarrow \cdots \rightarrow q_{i-1}=x_{2} \rightarrow q_{i}=x$. Thus there is an element $p_{k}=q_{k}$ in the saturated chain such that $p_{0}=q_{0}, p_{1}=q_{1}, \ldots, p_{k}=q_{k}$ and $p_{k+1} \neq q_{k+1}$. Hence $p_{k}$ is covered by two elements, a contradiction.

The case for $v$-interval of type (V2) is proved in a similar way.

The following corollary immediately follows from the above Proposition.

Corollary 7.5. Let $P$ be a minuscule heap over $\Gamma$. If $P$ contains a $v$-interval of type (Vi) $(1 \leq i \leq 3)$ for some $v \in N(\Gamma)$ then any $u$-interval is of type (Vi) for any $u \in N(\Gamma)$.

We should make a remark about minuscule heaps $(P, \leq, \phi)$ with supp $P \neq \Gamma$. Let $v \in N(\Gamma) \backslash \operatorname{supp} P$. If we choose a graph automorphism $\Phi: \Gamma \rightarrow \Gamma$ such that $\Phi(v)=0$ then we can regard $(P, \leq, \Phi \circ \phi)$ as a minuscule heap over the Dynkin diagram $\Gamma^{\prime}$ of type $A_{n}$. And the minuscule heaps over $\Gamma^{\prime}$ are already classified. To summarize, we now know the following:,

- If $P$ is a totally ordered set then $P$ is either of type (T1) or (T2).

- If $P$ is not a totally ordered set with supp $P \neq \Gamma$ then $P$ is a minuscule heap over a Dynkin diagram of type $A$.

The remaining case is that when $P$ is not totally ordered and $\operatorname{supp} P=\Gamma$. We know that any $v$-interval of $P$ is a diamond for any $v \in N(\Gamma)$. In $\S 9$ we study such minuscule heaps. 

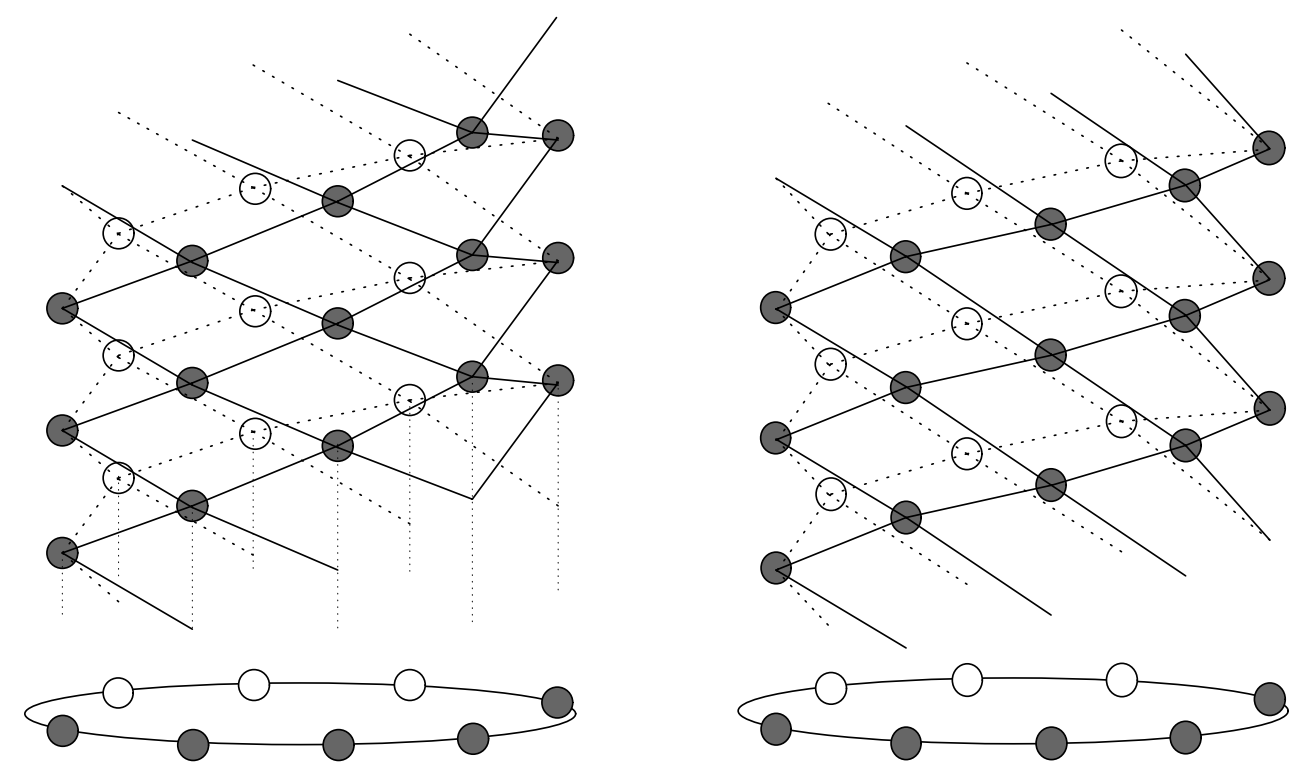

Figure 2: extended slant lattices $L_{3}$ (left figure) and $L_{4}$ (right figure) over $\Gamma\left(\tilde{A}_{7}\right)$

\section{An Extended Slant Lattice}

In this section we introduce the notion of extended slant lattices $L_{k}(1 \leq k \leq n)$ (see figure

2 ) which are $\Gamma$-labeled posets such that any minuscule heaps over $\Gamma\left(\tilde{A}_{n}\right)$ are isomorphic to those convex subsets.

We put $L_{0}:=\{(a, b) \in \mathbb{Z} \times \mathbb{Z} \mid a \equiv b(\bmod 2)\}$ and define a relation $\rightarrow$ on $L_{0}$ by $(v, m) \rightarrow(v, m)+(1,-1)$ or $(v, m) \rightarrow(v, m)+(-1,-1)$. Let $\leq$ be the reflective, transitive closure of $\rightarrow$. We note that $\left(L_{0}, \leq\right)$ is a poset. Then the following lemma is obtained immediately.

Lemma 8.1. Let $Q$ be an order ideal generated by $(0,0)$ of $L_{0}$. Then $Q=\{(a, b)|b \geq| a \mid\}$, where $\|$ is the absolute value symbol.

For $1 \leq k \leq n$, we define a map $\psi_{k}: \mathbb{Z} \times \mathbb{Z} \rightarrow \mathbb{Z} \times \mathbb{Z}$ by putting $\psi_{k}(v, m):=$ $(v+n+1, m-2 k+n+1)$. Then $\psi_{k}$ is a poset automorphism of $(\mathbb{Z} \times \mathbb{Z}, \leq)$. Let $G_{k}$ be the group generated by $\psi_{k}$. Then $G_{k}$ satisfies the conditions of Prop. 3.3. Hence we define a poset $L_{k}$ by putting

$$
L_{k}:=\left(\mathbb{Z} \times \mathbb{Z} / G_{k}, \leq_{L_{k}}\right)
$$

and call it an extended slant lattice. We note that the sum + , defined by putting $\overline{(v, m)}+$ $\overline{\left(u, m^{\prime}\right)}:=\overline{\left(v+u, m+m^{\prime}\right)}$ for $\overline{(v, m)}$ and $\overline{\left(u, m^{\prime}\right)}$, is well-defined on $L_{k} .\{(v, m) \in[0, n] \times$ $\mathbb{Z} \mid v \equiv m(\bmod 2)\}$ is a complete representative system of $L_{k}$. Thus we can define a $\operatorname{map} \phi: L_{k} \rightarrow[0, n]$ by putting $\phi(\overline{(v, m)}):=v$, where $0 \leq v \leq n$. Now we regard $L_{k}=\left(\mathbb{Z} \times \mathbb{Z} / G_{k}, \leq_{L_{k}}, \phi\right)$ as a $\Gamma$-labeled poset by this map. 
Let us define a binary relation $\leadsto$ by $\overline{(v, m)} \leadsto \overline{(v+1, m-1)}, \overline{(v, m)} \leadsto \overline{(v-1, m-1)}$ for $\overline{(v, m)}, \overline{(v+1, m-1)}, \overline{(v-1, m-1)} \in L_{k}$ and put $\preceq$ the reflective and transitive closure of $\leadsto$.

Lemma 8.2. $\preceq$ is equivalent to $\leq$.

Proof. Let $p, q$ be elements of $L_{k}$ which satisfy $p \preceq q$. Then we can take a sequence $p=$ $p_{0} \leadsto p_{1} \leadsto \cdots \leadsto p_{r}=q$. Now we have $p_{i}=p_{i-1}+\overline{( \pm 1,-1)}$. If we put $p_{i-1}=\overline{(v, m)}$ then we can write $p_{i}=\overline{(v \pm 1, m-1)}$. By the definition of $\leq$, we have $(v, m) \leq_{L_{0}}(v \pm 1, m-1)$. Thus we have $\overline{(v, m)} \leq_{L_{k}} \overline{(v \pm 1, m-1)}$. This implies $p \leq_{L_{k}} q$.

Conversely assume that $\overline{(v, m)} \leq_{L_{k}} \overline{(u, l)}$. Then we have $(v, m) \leq_{L_{0}}(u, l)+j(n+$ $1, n+1-2 k)$ for some $j \in \mathbb{Z}$. Let $p_{0}, p_{1}, \ldots, p_{r}$ be a saturated chain from $(v, m)$ to $\underline{(u+j}(n+1), l+j(n+1-2 k))$. Then we have $\overline{p_{0}} \leadsto \overline{p_{1}} \leadsto \cdots \leadsto \overline{p_{r}}$. Hence $\overline{(v, m)} \preceq$ $\overline{(u, l)}$.

Although the following proposition maybe seems to be obvious, it does not hold on $L_{1}, L_{n}$.

Proposition 8.3. For $2 \leq k \leq n-1, \overline{(v, m)} \in L_{k}$ covers $\overline{(v \pm 1, m+1)} \cdot \overline{(v, m)}$ is covered by $\overline{(v \pm 1, m-1)}$.

Proof. It is sufficient to show that $\leadsto$ is a cover relation on $L_{k}$. To do so, we show that $\overline{(a, b)}=s_{0} \leadsto s_{1} \leadsto \cdots \sim s_{r}=\overline{(a \pm 1, b-1)}$ implies $r=1$ by Lemma 3.2.

First we prove in the case $s_{r}=\overline{(a+1, b-1)}$. Put $\alpha:=\#\left\{1 \leq i \leq r \mid s_{i}=s_{i-1}+\right.$ $\overline{(1,-1)}\}$ and put $\beta:=\#\left\{1 \leq i \leq r \mid s_{i}=s_{i-1}+\overline{(-1,-1)}\right\}$. (We note that $\alpha+\beta=r$ and $\alpha, \beta \geq 0$.) Then we have $s_{r}=\overline{(a+\alpha-\beta, b-\alpha-\beta)}=\overline{(a+1, b-1)}$. This implies that

$$
\begin{aligned}
a+\alpha-\beta & =a+1+\gamma(n+1), \\
b-\alpha-\beta & =b-1+\gamma(n+1-2 k),
\end{aligned}
$$

for some $\gamma \in \mathbb{Z}$. The solution of above equations is $\alpha=1+k \gamma, \beta=\gamma(k-n-1)$. By the assumption, we have $2 \leq k \leq n-1$ and $\alpha \geq 0$. Thus, $\gamma \geq 0$. On the other hand, $k-n-1<0$ and $\beta \geq 0$. So, $\gamma \leq 0$. This implies $\gamma=0$. Then we have $\alpha=1$ and $\beta=0$. So we conclude that $r=\alpha+\beta=1+0=1$ holds.

By using a similar argument, we have $\alpha=0$ and $\beta=1$ from the case $s_{r}=\overline{(a-1, b-1)}$.

Remark 8.4. In the proof above, there exists a solution $\alpha=0, \beta=n$ for $s_{r}=$ $\overline{(a+1, b-1)}, k=1$ and $\gamma=-1$. Hence $\leadsto$ is not a covering relation on $L_{1}$.

Proposition 8.5. $L_{1}, L_{n}$ are totally ordered sets.

Proof. We give only proof for $L_{1}$. (We can similarly prove it for $L_{n}$.)

We know that $\{\overline{(v, m)} \mid 0 \leq v \leq n, m \equiv v(\bmod 2)\}$ is a complete representative system. For $\overline{(0,2 m)}$, we have $\overline{(0,2 m)}=\overline{(m(n+1), m(n+1))}$. So we can write $\overline{(i, i+2 m)}=$ $\overline{(i+m(n+1), i+m(n+1))}$ for $0 \leq i \leq n$. Thus $L_{1}$ is isomorphic to $\mathbb{Z}$ as poset. (We note that $-j$ is an element on $\mathbb{Z}$ corresponding to $\overline{(j, j)}$. For example, we have $\overline{(0,0)}>\overline{(1,1)}$ in the ordering $\leq$ on $L_{1}$.) 
Corollary 8.6. For $1 \leq k \leq n$, we have that (H1-1),(H1-2) and (H2a) hold on $L_{k}$, and their finite convex subsets are minuscule. Moreover their $v$-intervals are diamonds when $2 \leq k \leq n-1$.

Proof. By Propositions 8.3, 8.5, (H1-1) holds on $L_{k}$. By the definition of $\preceq$ and by the fact that (H1-2) holds on $L_{0}$, (H1-2) holds on $L_{k}$.

Next we show that (H2a) holds. If $k=1$ or $k=n$ then $P$ has the structure in Prop. 6.2. So (H2a) holds. By the definition of $L_{k}$, a $v$-interval is a diamond when $2 \leq k \leq n-1$. So (H2a) holds.

Thus their finite convex subsets are minuscule.

For a finite subset $Q$ of $L_{k}$ and $v \in N(\Gamma)$, we can define a unique maximal (resp. minimal) element $t_{v}$ (resp. $b_{v}$ ) of $Q_{v}$ since (H1-2) holds on $L_{k}$

Lemma 8.7. Let $y_{0} \in \mathbb{Z}$ and put $Q:=\left\{p \in L_{k} \mid p \leq \overline{\left(k, y_{0}\right)}\right\}$. Then we have

$$
Q=\left\{\overline{(a, b)} \mid 0 \leq a \leq k, b \geq y_{0}+k-a\right\} \cup\left\{\overline{(a, b)} \mid k<a \leq n, b \geq y_{0}+a-k\right\} .
$$

Let $x_{0} \in \mathbb{Z}$ and put $Q^{\prime}:=\left\{p \in L_{k} \mid p \geq \overline{\left(n+1-k, x_{0}\right)}\right\}$. Then we have

$$
\begin{aligned}
& Q^{\prime}=\{\overline{(a, b)} \mid 0 \leq a \leq n+1-k, b\left.\leq x_{0}+k+a\right\} \\
& \cup\left\{\overline{(a, b)} \mid n+1-k<a \leq n, b \leq x_{0}+2 n+2-k-a\right\} .
\end{aligned}
$$

Proof. We can take a complete representative system $\{\overline{(v, m)} \mid 0 \leq v \leq n, m \equiv v(\bmod 2)\}$. For $0 \leq a \leq n$, we have

$$
\begin{aligned}
& \overline{(a, b)} \leq \overline{\left(k, y_{0}\right)} \\
& \Longleftrightarrow \quad(a, b)+j(n+1, n+1-2 k) \leq\left(k, y_{0}\right) \exists j \in \mathbb{Z} \\
& \Longleftrightarrow \quad\left(a-k+j(n+1), b-y_{0}+j(n+1-2 k)\right) \leq(0,0) \exists j \in \mathbb{Z}
\end{aligned}
$$

(By Lemma 8.1)

$$
\Longleftrightarrow \quad b-y_{0}+j(n+1-2 k) \geq|a-k+j(n+1)| \exists j \in \mathbb{Z} \text {. }
$$

Assume $0 \leq a \leq k$. Then we have $a-k+j(n+1) \leq 0 \Longleftrightarrow j \leq 0$ or equivalently we have $a-k+j(n+1) \geq 0 \Longleftrightarrow j \geq 1$. For $j \leq 0$, we have

$$
\begin{aligned}
b & \geq y_{0}-j(n+1-2 k)-a+k-j(n+1) \\
& =y_{0}+k-a-2 j(n+1-k) .
\end{aligned}
$$

Hence the necessary and sufficient condition for $\left(a-k+j(n+1), b-y_{0}+k+j(n+1-2 k)\right) \leq$ $(0,0)$ (for some $j \leq 0)$ is

$$
b \geq y_{0}+k-a
$$

For $j \geq 1$, we have

$$
\begin{aligned}
b & \geq y_{0}-j(n+1-2 k)+a-k+j(n+1) \\
& =y_{0}+a-k+2 j k .
\end{aligned}
$$


Hence the necessary and sufficient condition for $\left(a-k+j(n+1), b-y_{0}+j(n+1-2 k)\right) \leq$ $(0,0)$ (for some $j \geq 1$ ) is

$$
b \geq y_{0}+a .
$$

Thus we have $b \geq y_{0}+k-a$. Assume $k<a \leq n$. We have $a-k+j(n+1) \geq 0 \Longleftrightarrow$ $j \geq 0$ or equivalently $a-k+j(n+1) \leq 0 \Longleftrightarrow j \leq-1$. For $j \geq 0$ we have

$$
b \geq y_{0}+a-k .
$$

For $j \leq-1$ we have

$$
b \geq y_{0}-a+2 n+2-k .
$$

On the other hand, we have $y_{0}-a+2 n+2-k \geq y_{0}+a-k$. Thus we have $b \geq y_{0}+a-k$.

We can prove for the case for $Q^{\prime}$ in a similar way.

Let $x_{0}, y_{0} \in \mathbb{Z}$ satisfying $x_{0} \geq y_{0}$. For $v \in N(\Gamma)(=[0, n])$, we define $y_{v}$ by putting $y_{v}:=y_{0}+k-v$ if $0 \leq v \leq k$ and $y_{v}:=y_{0}+a-k$ if $k<v \leq n$. We define $x_{v}$ by putting $x_{v}:=x_{0}+k+v$ if $0 \leq v \leq n+1-k$ and $x_{v}:=x_{0}+2 n+2-k-v$ if $n+1-k<v \leq n$. Then we have the following,

\section{Proposition 8.8.}

$$
\left[\overline{\left(n+1-k, x_{0}+n+1-k\right)}, \overline{\left(k, y_{0}-k\right)}\right]=\left\{(v, m) \in L_{k} \mid y_{v} \leq m \leq x_{v}\right\}
$$

Proof. It is obvious if we take $Q \cap Q^{\prime}$ in Lemma 8.7.

Proposition 8.9. Let $2 \leq k \leq n-1$ and let $Q$ be a finite subposet of $L_{k}$ satisfying $\operatorname{supp} Q=\Gamma$. For $v \in N(\Gamma)$, put $b_{v}:=\overline{\left(v, x_{v}\right)}$ and $t_{v}:=\overline{\left(v, y_{v}\right)}$, where $t_{v}\left(\right.$ resp $\left.b_{v}\right)$ is a maximal (resp. minimal) element of $P_{v}$.

$Q$ is a convex subset if and only if $Q$ satisfies the followings,

- $Q_{v}=\left\{\overline{(v, m)} \in L_{k} \mid y_{v} \leq m \leq x_{v}\right\}$,

- $t_{v-1}, t_{v}$ are a covering pair,

- $b_{v-1}, b_{v}$ are a covering pair,

for each $1 \leq v \leq n+1$.

Proof. Let $Q$ be a convex subset. Since (H1-2) holds on $L_{k}$, we have $Q_{v}=\{\overline{(v, m)} \in$ $\left.L_{k} \mid y_{v} \leq m \leq x_{v}\right\}$.

Assume that $\overline{(v-1, m)}, \overline{(v, l)} \in Q$ for some $1 \leq v \leq n$. If we have $m<l$ then there exists a saturated chain $\overline{(v, l)} \rightarrow \overline{(v-1, l-1)} \rightarrow \overline{(v, l-2)} \rightarrow \cdots \rightarrow \overline{(v, m-1)} \rightarrow$ $\overline{(v-1, m)}$ on $L_{k}$. Since $Q$ is a convex subset, this saturated chain is contained in $Q$. This implies that we have $-1 \leq y_{v-1}-y_{v}$. Next we assume $m>l$. Then we have $y_{v-1}-y_{v} \leq 1$ by a similar argument. So we have $-1 \leq y_{v-1}-y_{v} \leq 1$. This implies that $t_{v-1}$ and $t_{v}$ are a covering pair. By a similar argument, $b_{v-1}$ and $b_{v}$ are a covering pair. 
$\overline{(0, m)}=\overline{(n+1, m-2 k+n+1)}$ implies that we have $\left|y_{n}-y_{0}+2 k-n-1\right|=\mid x_{n}-$ $x_{0}+2 k-n-1 \mid=1$. This implies that $t_{0}$ and $t_{n}$ (or $b_{0}$ and $b_{n}$ ) are a covering pair.

Conversely let $Q$ be a subset satisfying the condition. We claim that $Q$ is obtained from $\left[\overline{\left(n+1-k, x_{0}+n+1-k\right)}, \overline{\left(k, y_{0}-k\right)}\right]$ by deleting its maximal or minimal elements. Thus it follows that $P$ is a convex subset by Propositions 3.4, 3.5.

For $1 \leq v \leq n$ if we have $t_{v-1} \leftarrow t_{v}$ and $t_{v} \rightarrow t_{v+1}$ then we construct another poset $Q^{\prime}=Q_{v} \cup\left\{\overline{\left(v, y_{v}-2\right)}\right\}$. We note that $Q^{\prime}$ satisfies the condition. We regard $Q$ as $Q^{\prime} \backslash\left\{\overline{\left(c, y_{v}-2\right)}\right\}$. We also note that $Q_{0}=Q_{0}^{\prime}$. By repeating this operation, we obtain a poset $Q^{\prime}$ such that $t_{0} \rightarrow t_{1} \rightarrow \cdots \rightarrow t_{k}$ and $t_{k} \leftarrow t_{k+1} \leftarrow \cdots \leftarrow t_{n} \leftarrow t_{n+1}=t_{0}$. For $1 \leq v \leq n$ if we have $b_{v-1} \rightarrow b_{v}$ and $b_{v} \leftarrow b_{v+1}$ then we obtain another poset $Q^{\prime} \cup\left\{\overline{\left(v, x_{v}+2\right)}\right\}$. By repeating this operation, we obtain a poset $Q^{\prime}$ such that $b_{0} \leftarrow$ $b_{1} \leftarrow \cdots \leftarrow b_{n+1-k}$ and $b_{n+1-k} \rightarrow \cdots \rightarrow b_{n} \rightarrow b_{n+1}=b_{0}$. By Prop. 8.8, we have $Q^{\prime}=\left[\overline{\left(n+1-k, x_{0}+n+1-k\right)}, \overline{\left(k, y_{0}-k\right)}\right]$. In particular $Q^{\prime}$ is a convex subset. Thus $Q$ is a convex subset.

\section{Classification of Minuscule Heaps over $\Gamma(\tilde{A})$}

In this section, we classify the minuscule heaps over $\Gamma=\Gamma\left(\tilde{A}_{n}\right)$ (Theorem 9.8). In previous sections, we classified some minuscule heaps. The remaining case is that of minuscule heap $P$ over $\Gamma$ such that $n \geq 2, \operatorname{supp} P=\Gamma$, and its $v$-intervals are of type (V3).

Let $P$ be a minuscule heap over $\Gamma$ which is not totally ordered. First we observe the structure of $P_{i} \cup P_{i+1}$, where $P_{i}=\{p \in P \mid \phi(p)=i\}$. By (H1-2), $P_{i} \cup P_{i+1}$ is a totally ordered set. We denote the number of element of $P_{i}$ (resp. $P_{i+1}$ ) by $r_{i}$ (resp. $\left.r_{i+1}\right)$. Therefore there are $\left(r_{i}-1\right) i$-intervals in $P_{i} \cup P_{i+1}$. We note that each $i$-interval contains one element of $P_{i+1}$. Thus we have $r_{i+1} \geq r_{i}-1$. By a similar argument, we have $r_{i} \geq r_{i+1}-1$. Hence we have $r_{i}+1 \geq r_{i+1} \geq r_{i}-1$.

Let us assume $r_{i+1}=r_{i}-1$. Now there are $r_{i}-1\left(=r_{i+1}\right) i$-intervals. Each $i$-interval contains one element of $P_{i+1}$. Since an $i$-interval is a diamond, if we take an element of $P_{i}$ and an element of $P_{i+1}$ which is contained in the interval then they are a covering pair. Let $p_{1}, p_{2}, \ldots, p_{r_{i}}$ be the elements of $P_{i}$ in the increasing order and let $q_{1}, q_{2}, \ldots, q_{r_{i+1}}$ be the elements of $P_{i+1}$ in the increasing order. By the argument above, we have

$$
p_{1} \rightarrow q_{1} \rightarrow p_{2} \rightarrow q_{2} \rightarrow \cdots \rightarrow q_{r_{i+1}} \rightarrow p_{r_{i}}
$$

By a similar argument if we assume $r_{i+1}=r_{i}+1$ then we have

$$
q_{1} \rightarrow p_{1} \rightarrow q_{2} \rightarrow p_{2} \rightarrow \cdots \rightarrow p_{r_{i}} \rightarrow q_{r_{i+1}}
$$

Next let us assume $r_{i}=r_{i+1}$. By a similar argument, we have either

$$
q_{1} \rightarrow p_{1} \rightarrow q_{2} \rightarrow p_{2} \rightarrow \cdots \rightarrow q_{r_{i+1}}<p_{r_{i}}
$$

or

$$
p_{1}<q_{1} \rightarrow p_{2} \rightarrow q_{2} \rightarrow \cdots \rightarrow q_{r_{i}} \rightarrow p_{r_{i}} \rightarrow q_{r_{i+1}}
$$


In the former case, $\left[p_{r_{i}-1}, p_{r_{i}}\right]$ is an $i$-interval. Thus we have $q_{r_{i+1}} \rightarrow p_{r_{i}}$. In the latter case, we have $p_{1} \rightarrow q_{1}$. At this point, we have completed to determine the structure of $P_{i} \cup P_{i+1}$. We record these results in the following corollaries 9.1, 9.2,

Corollary 9.1. Let $P$ be a minuscule heap with $\operatorname{supp} P=\Gamma$ such that $P$ is not a totally ordered set. Then $t_{i}, t_{i+1}\left(b_{i}, b_{i+1}\right)$ are a covering pair for each $i \in N(\Gamma)$.

Corollary 9.2. For $v \in N(\Gamma)$, put $r_{v}:=\# P_{v}$. Then we have,

- " $r_{i+1}=r_{i}$ " is equivalent to either " $t_{i} \rightarrow t_{i+1}$ and $b_{i} \rightarrow b_{i+1}$ " or " $t_{i} \leftarrow t_{i+1}$ and $b_{i} \leftarrow b_{i+1} "$,

- $r_{i+1}=$ " $r_{i}+1$ " is equivalent to " $t_{i} \rightarrow t_{i+1}$ and $b_{i} \leftarrow b_{i+1}$ ",

- $r_{i+1}=$ " $r_{i}-1$ " is equivalent to " $t_{i} \leftarrow t_{i+1}$ and $b_{i} \rightarrow b_{i+1}$ ".

We call the number of covering pairs satisfying $t_{v} \rightarrow t_{v+1}(1 \leq v \leq n+1)$ the gradient of $P$.

Proposition 9.3. Let $P$ be a minuscule heap over $\Gamma$ which is not totally ordered and supp $=\Gamma$. Let $k$ be a gradient of $k$. Then we have $2 \leq k \leq n-1$.

Proof. If $k=1$ then there is a unique covering pair $t_{i-1}, t_{i}$ such that $t_{i-1} \rightarrow t_{i}$. On the other hand, we have $t_{i-1} \rightarrow t_{i-2} \rightarrow \ldots t_{i+1} \rightarrow t_{i}$. It contradicts that $t_{i-1}, t_{i}$ are a covering pair.

We can prove, for the case $k=n$, by using a similar argument.

Let $P$ be a not totally ordered minuscule heap with supp $P=\Gamma$ and let $k$ be the gradient of $P$. Define a map $\nu: P \rightarrow L_{k}$ by putting,

$$
\begin{gathered}
\nu\left(t_{0}\right):=\overline{(0,0)}, \\
\nu\left(t_{i}\right):= \begin{cases}\nu\left(t_{i-1}\right)+\overline{(1,-1)} & \text { if } t_{i-1} \rightarrow t_{i}, \\
\nu\left(t_{i-1}\right)+\overline{(1,1)} & \text { if } t_{i-1} \leftarrow t_{i},\end{cases}
\end{gathered}
$$

and if $p$ is $j$-th largest element in $P_{v}$ then

$$
\nu(p):=\nu\left(t_{v}\right)+\overline{(0,2 j-2)} .
$$

We note that $\nu$ is an injection.

Proposition 9.4. Let $t_{n}$ (resp. $t_{0}$ ) be the unique maximal element of $P_{n}$ (resp. $\left.P_{0}\right)$. If $t_{n} \rightarrow t_{0}$ then we have $\nu\left(t_{n}\right) \rightarrow \nu\left(t_{0}\right)$. If $t_{n} \leftarrow t_{0}$ then we have $\nu\left(t_{n}\right) \leftarrow \nu\left(t_{0}\right)$.

Proof. Assume $t_{n} \rightarrow t_{0}$. Now we have $\#\left\{1 \leq i \leq n \mid t_{i-1} \rightarrow t_{i}\right\}=k-1$. Thus,

$$
\begin{aligned}
\nu\left(t_{n}\right) & =\nu\left(t_{0}\right)+(k-1) \overline{(1,-1)}+(n-k+1) \overline{(1,1)} \\
& =\nu\left(t_{0}\right)+\overline{(n, n-2 k+2)} \\
& =\nu\left(t_{0}\right)+\overline{(-1,1)} \\
& \rightarrow \nu\left(t_{0}\right) .
\end{aligned}
$$


Assume $t_{n} \leftarrow t_{0}$. Now we have $\#\left\{1 \leq i \leq n \mid t_{i-1} \rightarrow t_{i}\right\}=k$. Thus we have

$$
\begin{aligned}
\nu\left(t_{n}\right) & =\nu\left(t_{0}\right)+\overline{(\overline{1,-1)}}+(n-k) \overline{(1,1)} \\
& =\nu\left(t_{0}\right)+\overline{(n, n-2 k)} \\
& =\nu\left(t_{0}\right)+\overline{(-1,-1)} \\
& \leftarrow \nu\left(t_{0}\right) .
\end{aligned}
$$

We note that $\nu$ preserves the cover-relations on $t_{0}, t_{1}, \ldots, t_{n}$.

Proposition 9.5. Let $P$ be a minuscule heap over $\Gamma$ which is not totally ordered and let $k$ be the gradient of $P$. Let $\nu$ be a map defined as above. Then $\operatorname{Im} \nu$ is a convex subset in $L_{k}$.

Proof. Put $Q:=\operatorname{Im} \nu$. For $0 \leq v \leq n, Q_{v}$ satisfies the condition in Prop. 8.9 by the definition of $\nu$. Let $t_{v}^{\prime}$ be a maximal element of $Q_{v}$.

Then $t_{v}^{\prime}$ and $t_{v+1}^{\prime}$ are a covering pair since $\nu$ preserves the cover-relations on $t_{0}, t_{1}, \ldots, t_{n}$. Finally we show that $b_{v}^{\prime}$ and $b_{v+1}^{\prime}$ are a covering pair, where $b_{v}^{\prime}$ is a minimal element of $Q_{v}$. Assume $t_{v} \rightarrow t_{v+1}$. By Corollary 9.2, $b_{v} \rightarrow b_{v+1}$ implies $r_{v}=r_{v+1}$. Thus we have $b_{v+1}^{\prime}=\nu\left(b_{v+1}\right)=\nu\left(t_{v+1}\right)+\overline{\left(0,2\left(r_{v+1}-1\right)\right)}=\nu\left(t_{v}\right)+\overline{(1,-1)}+\overline{\left(0,2\left(r_{v}-1\right)\right)}=$ $\nu\left(b_{v}\right)+\overline{(1,-1)} \leftarrow \nu\left(b_{v}\right)=b_{v}^{\prime} . \quad b_{v} \leftarrow b_{v+1}$ implies $r_{v+1}=r_{v}+1$. Now we have $b_{v+1}^{\prime}=\nu\left(b_{v+1}\right)=\nu\left(t_{v}\right)+\overline{\left(0,2\left(r_{v+1}-1\right)\right)}=\nu\left(t_{v+1}\right)+\overline{\left(0,2 r_{v}\right)}=\nu\left(t_{v}\right)+\overline{(1,-1)}+\overline{\left(0,2 r_{v}\right)}=$ $\nu\left(t_{v}\right)+\overline{(1,1)}+\overline{\left(0,2\left(r_{v}-1\right)\right)}=\nu\left(b_{v}\right)+\overline{(1,1)} \rightarrow \nu\left(b_{v}\right)=b_{v}^{\prime}$.

We can prove in the case $t_{v} \leftarrow t_{v+1}$ by a similar argument.

Proposition 9.6. Let $P$ be a minuscule heap over $\Gamma$ which is not totally ordered and let $k$ be the gradient of $P$. Then $P$ is isomorphic to $\operatorname{Im} \nu$ as a $\Gamma$-labeled poset.

Proof. Let $p, q$ be elements of $P$ which satisfy $p \rightarrow q$. Then we show that $\nu(p)<\nu(q)$. Assume $p \in P_{v}$ and $q \in P_{v+1}$. (We can similarly argue in the case $p \in P_{v+1}, q \in P_{v}$.) And we assume $t_{v} \rightarrow t_{v+1}$. If $p$ is the $r$-th element in $P_{v}$ by an increasing order then $q$ is the $r$-th element in $P_{v+1}$ by an argument in the preceding Corollary 9.1. Thus we have $\nu(q)=\nu\left(t_{v+1}\right)+\overline{(0,2(r-1))}=\nu\left(t_{v}\right)+\overline{(1,-1)}+\overline{(0,2(r-1))}=\nu(p)+\overline{(1,-1)}+$ $\overline{(0,2(r-1))}=\nu(p)+\overline{(1,-1)} \leftarrow \nu(p)$. Hence we have $\nu(q) \geq \nu(p)$. Assume $t_{v} \leftarrow t_{v+1}$. If $p$ is the $r$-th element in $P_{v}$ then $q$ is the $(r-1)$-th element in $P_{v+1}$. Thus we have $\nu(q)=$ $\nu\left(t_{v+1}\right)+\overline{(0,2(r-2))}=\nu\left(t_{v}\right)+\overline{(1,1)}+\overline{(0,2(r-2))}=\nu\left(t_{v}\right)+\overline{(1,-1)}+\overline{(0,2(r-1))}=$ $\nu(p)+\overline{(1,-1)} \leftarrow \nu(p)$. So we have $\nu(q) \geq \nu(p)$.

The arguments above show that $p \leq q$ implies $\nu(p) \leq \nu(q)$.

Conversely we assume $\nu(p) \rightarrow \nu(q)$. Since $\operatorname{Im} \nu$ is a convex subset, we have either $\nu(q)=\nu(p)+\overline{(1,-1)}$ or $\nu(q)=\nu(p)+\overline{(-1,-1)}$. Assume that $p \in P_{v}, q \in P_{v+1}$. (We can prove in the case $p \in P_{v+1}, q \in P_{v}$ by a similar argument.) If $t_{v} \rightarrow t_{v+1}$ then we have $\nu(q)=\nu(p)+\overline{(1,-1)}=\nu\left(t_{v}\right)+\overline{(0,2(r-1))}+\overline{(1,-1)}=\nu\left(t_{v}\right)+\overline{(1,-1)}+\overline{(0,2(r-1))}=$ $\nu\left(t_{v+1}\right)+\overline{(0,2(r-1))}$. Thus $q$ is the $r$-th element in $P_{v+1}$. By an argument in the preceding Corollary 9.1, we have $p \rightarrow q$. If $t_{v} \leftarrow t_{v+1}$ then $q$ is the $(r-1)$-th element 
in $P_{v+1}$ by a similar argument. Thus we have $p \rightarrow q$. Now $\operatorname{Im} \nu$ is a convex subset. Hence if $\nu(p) \leq \nu(q)$ then we can take a saturated chain from $\nu(p)$ to $\nu(q)$. Thus we have $p \leq q$.

By Propositions 9.5, 9.6, it follows that

Corollary 9.7. Let $P$ be a minuscule heap with gradient $k(2 \leq k \leq n-1)$ which is not totally ordered. Then $P$ is isomorphic to a convex subset of $L_{k}$.

Our main theorem gives the converse claim (Theorem 9.8).

In fact, a totally ordered minuscule heap is also isomorphic to a convex subset of $L_{k}$ for $k=1, n$. Let $Q$ be a totally ordered minuscule heap of type (T1) (resp. (T2)). Define a map $\nu: Q \rightarrow L_{n}$ by the following. If the label of a maximal element of $Q$ is $i$ then $\nu(q)=\overline{(i-j+1, i-j+1)}$ (resp. $\overline{(i+j-1, i-j+1)}$ where $q$ is the $j$-th element in $Q$ by an increasing ordering. It is obvious that $Q$ is isomorphic to $\operatorname{Im} \nu$ as a $\Gamma$-labeled poset. Now $\operatorname{Im} Q$ is a convex subset of $L_{n}$ (resp. $L_{1}$ ).

We note that a minuscule heap with supp $P \neq \Gamma$ can be identified with a minuscule heap over a Dynkin diagram of type $A$. Then it is isomorphic to a convex subset of $L_{k}$ for some $1 \leq k \leq n$.

Theorem 9.8. Let $n \geq 2$. Let $\Gamma$ be a Dynkin diagram of type $\tilde{A}_{n}$. A minuscule heap $P$ over $\Gamma$ is isomorphic to a convex subset of $L_{k}$, where $1 \leq k \leq n$ is determined by $P$. Conversely a finite convex subset of $L_{k}$ is a minuscule heap.

Remark 9.9. If a minuscule heap $P$ satisfies supp $P \neq \Gamma$ then $k$ in Theorem 9.8 is not unique.

Acknowledgments. The author would like to thank Professors I. Terada and K. Koike for helpful discussions.

\section{References}

[1] S.Billey, W.Jockusch and R.Stanley, Some combinatorial properties of Schubert polynomials, J. Algebraic Combinatorics, 2, 1993, 345-374

[2] A. Björner and F. Brenti, Affine Permutations of Type A, The electronic journal of combinatorics 3 (2) (1996), \#R18.

[3] V. V. Deodhar, Some Characterizations of Bruhat Ordering on a Coxeter Group and Determination of the Relative Möbius Function, Inventiones mathematicae 39, 1997, 187-198

[4] C. K. Fan, A Hecke Algebra Quotient and Properties of Commutative Elements of a Weyl Group, Ph.D. thesis, MIT, 1995.

[5] R. M. Green, On 321-avoiding permutations in affine Weyl groups, Journal of Algebraic Combinatorics, 15, 2002, 241-252. 
[6] HAGIWARA Manabu, Minuscule heaps over simply-laced, star-shaped Dynkin diagrams, preprint.

[7] J. Humphreys, "Reflection groups and Coxeter groups," Cambridge Univ. Press, 1990.

[8] V. G. Kac, "Infinite Dimensional Lie Algebras," Cambridge Univ. Press, Cambridge, 1990.

[9] Robert A. Proctor, Dynkin Diagram Classification of $\lambda$-Minuscule Bruhat Lattices and of d-Complete Posets, Journal of Algebraic Combinatorics, 9, 1999, 61-94.

[10] Robert A. Proctor, Minuscule elements of Weyl groups, the numbers game, and d-complete posets, J.Algebra, 213, 1999, 272-303

[11] John R. Stembridge, Minuscule Elements of Weyl Groups, J. Algebra, 235, 2001, 722-743.

[12] John R. Stembridge, On the Fully Commutative Elements of Coxeter Groups, J. Algebraic Combinatorics, 5, 1996, 353-385. 